\title{
EVOLUCIÓN BIOGEOGRÁFICA DE LOS PACHYDEMINAE PALEÁRTICOS (COLEOPTERA, SCARABAEOIDEA) MEDIANTE ANÁLISIS DE DISPERSIÓN-VICARIANZA
}

\author{
I. Sanmartín
}

\begin{abstract}
RESUMEN
En este trabajo, se reconstruye la historia biogeográfica de la subfamilia Pachydeminae Reitter, 1902 (Coleoptera, Scarabaeoidea, Melolonthidae) en el Paleártico occidental, utilizando el análisis de dispersión-vicarianza (DIVA). Este método de análisis biogeográfico reconstruye las distribuciones ancestrales en la filogenia de acuerdo con un simple modelo vicariante, pero al mismo tiempo permite considerar otros procesos como dispersión y extinción en la reconstrucción biogeográfica. Al contrario que otros métodos, no restringe las relaciones entre áreas a un modelo jerárquico por lo que puede utilizarse para reconstruir relaciones reticuladas. La reconstrucción óptima postulada por DIVA indica que el ancestro de los Pachydeminae Paleárticos probablemente se originó en la región suroriental del Mediterráneo, incluyendo el Norte de África, Oriente Medio, la Meseta Iraní, y los Balcanes/Anatolia. Durante el Oligoceno-Mioceno, la colisión de las Placas Africana, Arábiga, y Eurasiática dio lugar a la aparición de sucesivas barreras geográficas (e. g., el Mar Rojo, las montañas del Zagros) que dividieron el área ancestral de Pachydeminae, y dieron lugar a varios géneros por vicarianza (e. g., Pachydema Castelnau, 1832). El Oriente Medio y la Meseta Iraní habrían actuado como centros de diversificación en la evolución de la subfamilia: muchos de los géneros de Pachydeminae se originaron en estas regiones por especiación simpátrica (e. g., Otoclinius Brenske, 1896). La distribución de los Pachydeminae en el Mediterráneo occidental, en cambio, es el resultado de una dispersión posterior. El ancestro de los géneros ibéricos Ceramida Baraud, 1987 y Elaphocera Gené, 1836 probablemente se dispersó desde el Oriente Medio hacia la Península Ibérica a través del Norte de África y el Estrecho de Gibraltar. Esta dispersión pudo tener lugar a finales del Mioceno, durante la "crisis de salinidad" del Mesiniense, cuando la desecación parcial del Mar Rojo y el Mediterráneo permitió la dispersión de linajes asiáticos al Norte de África y la Península Ibérica. La evolución posterior de los géneros Elaphocera y Ceramida implicó la existencia de varios eventos de dispersión y vicarianza entre el Mediterráneo occidental y oriental, y entre la Península Ibérica y el Norte de África.
\end{abstract}

Palabras clave: Coleoptera, Scarabaeoidea, Melolonthidae, historia biogeográfica reticulada, análisis de dispersión-vicarianza, Cuenca Mediterránea, Mar Rojo, Mesiniense, dispersión norteafricana.

\section{ABSTRACT \\ Biogeographic history of the Palearctic Pachydeminae (Coleoptera, Scarabaeoidea) inferred by dispersal-vicariance analysis}

The beetle subfamily Pachydeminae Reitter, 1902 is one of the least-known subfamilies of Melolonthidae or "leaf-chafers" (Coleoptera, Scarabaeoidea). Some species of Pachydeminae have recently been described as agricultural pests of olive trees. The Pachydeminae are distributed in all major zoogeographical regions (except Australia and India) but their distribution is very disjunct. In the Palearctic region, they are distributed across southern Eurasia from the Canary Islands to China, including southern Europe (except France and Italy), North Africa, Asia Minor, Middle East, Iran-Afghanistan, Caucasus, and Central Asia. The majority of species occur in the southwestern Palearctic, with only a few species in

* Dirección: Department of Systematic Zoology, Evolutionary Biology Centre, Uppsala University. Norbyvägen 18D, SE-752 36, Uppsala, Sweden. email: isabel.sanmartin@ebc.uu.se 
China. As in the rest of Melolonthidae subfamilies (Browne \& Scholtz, 1999), phylogenetic relationships within the Pachydeminae are poorly resolved. Recently, Sanmartín \& Martín-Piera, (2003) reviewed the systematics of the Palearctic genera, and proposed the first phylogenetic hypothesis within the subfamily.

This study summarizes the conclusions of Sanmartín (1998) and Sanmartín (2003), which reconstructed the biogeographic history of the subfamily Pachydeminae in the Palearctic region using dispersalvicariance analysis (DIVA, Ronquist, 1996, 1997). This method reconstructs the ancestral distribution in a given phylogeny based on a vicariance model, while allowing dispersal and extinction to occur. Unlike other methods, DIVA does not enforce area relationships to conform to a hierarchical "area cladogram" so it can be used to reconstruct "reticulate" biogeographic scenarios. DIVA optimal reconstructions suggest that the ancestor of Pachydeminae was originally present in the south-eastern Mediterranean region, including North Africa, the Middle East, the Iranian Plateau, and the Balkans/Anatolian region. During the Oligocene-Miocene, the collision between the Arabian, African, and Eurasian Plates resulted in the appearance of consecutive dispersal barriers (e.g., the Red Sea, the Zagros Mountains). This geographic division was followed by fragmentation (vicariance) of the ancestral biota, giving rise to several disjunct genera (Pachydema Castelnau, 1832, Hemictenius Reitter, 1897). The Middle East region and the Iranian Plateau acted as centers of diversification during the evolution of the subfamily: many of the least speciose genera of Pachydeminae originated within these two regions by sympatric speciation (Otoclinius Brenske, 1896). In contrast, the presence of Pachydeminae in the Western Mediterranean region (Iberian Peninsula and southwestern Mediterranean Islands) is the result of a more recent dispersal event. The ancestor of the Iberian genera Ceramida Baraud, 1987 and Elaphocera Gené, 1836 probably dispersed from the Middle East to the Iberian Peninsula across North Africa and the Gibraltar Strait. This dispersal could have taken place during the "Messinian salinity crisis" at the end of the Miocene, when the Red Sea and the Mediterranean partially dried-up, allowing a short period of biotic dispersal between West Asia, North Africa, and the Iberian Peninsula. The subsequent evolution of Ceramida and Elaphocera seem to have involved repeated vicariance events between the East and West Mediterranean, and between the Iberian Peninsula and North Africa. In the Iberian Peninsula, the two genera are geographically segregated: most species of Ceramida are found in the southwestern region, whereas Elaphocera is generally restricted to the southeastern Iberian Peninsula.

Key-Words: Coleoptera, Scarabaeoidea, Melolonthidae, reticulate biogeographic history, dispersalvicariance analysis, Mediterranean Basin, Red Sea, Messinian, North-African dispersal.

\section{Introducción}

En la reconstrucción de la historia biogeográfica de cualquier grupo de organismos, es necesario tener en cuenta dos procesos biogeográficos esenciales: dispersión y vicarianza. Ambos constituyen mecanismos históricos alternativos para explicar las disyunciones geográficas (i.e., distribuciones espacialmente discontinuas). La hipótesis "dispersionista" postula que el ancestro del grupo se originó en un área ancestral o "centro de origen", desde donde se dispersó a otras áreas atravesando barreras geográficas "preexistentes" (e. g., una cadena montañosa). La hipótesis "vicariante", en cambio, defiende que el ancestro del grupo ocupaba un área ancestral continua que se dividió sucesivamente en áreas menores por la aparición de nuevas barreras geográficas (e. g., la formación de una cuenca marina). Cada una de estas divisiones geográficas fue seguida por la fragmentación de la biota ancestral en sucesivos fenómenos de vicarianza. En ambos casos, el resultado final es el aislamiento espacial de una población por una barrera geográfica y la consiguiente diferenciación de un nuevo taxón por especiación alopátrica. Sin embargo, mientras que en la dispersión, la barrera es más antigua que la disyunción geográfica, en la explicación vicariante la aparición de la barrera geográfica es responsable de la disyunción y subsiguiente especiación, por lo que no puede se más antigua que ésta.

La mayor parte de los métodos actuales de análisis biogeográfico (Nelson \& Platnick, 1981; Brooks, 1990; Page, 1994) están basados en el modelo vicariante (el modelo "nulo" en biogeografía histórica) porque las hipótesis dispersionistas son, en principio, irrefutables: cualquier patrón de distribución puede ser explicado sólo con invocar suficientes fenómenos de dispersión (Morrone \& Crisci, 1995). Estos métodos consideran la dispersión como un fenómeno al azar, que permite explicar la evolución de cada organismo de forma independiente de otros organismos y de las áreas que ocupan, pero no la existencia de patrones generales de distribución. La biota de un área es por tanto la suma de las dispersiones de distintos linajes, cada uno con su propia historia biogeográfica. El mode- 
lo vicariante, en cambio, propugna la evolución conjunta de organismos y áreas: si dos o más grupos de organismos muestran en sus filogenias el mismo patrón de distribución geográfica, es posible postular que estos han compartido una historia biogeográfica común. Por tanto, es posible reconstruir la secuencia de fenómenos de vicarianza a partir de las filogenias de los organismos, sustituyendo en el cladograma el nombre de los organismos por las áreas que éstos ocupan. El resultado es el "cladograma general de áreas", que supuestamente refleja la relación entre las biotas de las áreas analizadas. Esta es la base de los que se ha llamado la "Escuela Biogeográfica Cladística" (Nelson \& Platnick, 1981; Humphries, 1992; Morrone \& Crisci, 1995).

Sin embargo, existen varios problemas a este planteamiento. No todas las especies responden igual al mismo evento vicariante, y eventos vicariantes de diferentes edades pueden aislar las mismas áreas ("pseudocongruencia"). Como resultado la biota de un área puede estar formada por componentes con historias biogeográficas distintas (Cracraft, 1989; Ronquist, 1997; Sanmartín et al., 2001). Por otro lado, mientras que los organismos presentan una genealogía ramificada (filogenia), las áreas no están necesariamente sujetas a una cladogénesis jerárquica (Ronquist, 1997). La configuración entre áreas puede cambiar con el tiempo, a medida que las áreas se crean, fragmentan, y colisionan. Procesos geológicos como la colisión entre dos placas continentales o el retroceso de un mar epicontinental que antes separaba dos regiones (e. g., el estrecho de Turgai entre Europa y Asia en el Terciario) favorecen la dispersión biótica ("difusión") entre áreas. Esto puede ser seguido por la aparición de una nueva barrera geográfica que divide de nuevo la biota ancestral por vicarianza (e. g., la elevación de los Urales entre Europa y Asia en el Oligoceno). Un ejemplo de este tipo de escenarios, en el que fenómenos de dispersión y vicarianza se suceden entre las mismas áreas, es la Región Holártica. Las reconstrucciones paleogeográficas del Hemisferio Norte muestran que las regiones holárticas estuvieron unidas en distintas configuraciones a través del tiempo (Smith et al., 1994). Este tipo de escenario, en el que las relaciones entre áreas no siguen un patrón jerárquico sino que presentan relaciones reticuladas ("hibridación entre áreas"), no puede ser representado mediante un cladograma de áreas (Sanmartín et al., 2001).

Otro problema del modelo vicariante es que ignora el papel de la dispersión como responsable de la distribución geográfica de los organismos. El modelo vicariante clásico asume que la distribución geográfica es el resultado de la aparición sucesiva de barreras dispersivas que dividen una biota ancestral, seguido de especiación alopátrica. La dispersión no se considera en la reconstrucción excepto para explicar el origen de la distribución ancestral continua, es decir, todos los eventos de dispersión ocurrieron antes de la primera cladogénesis. Durante el análisis biogeográfico, la dispersión sólo se considera a posteriori a la hora de interpretar la congruencia entre el cladograma de áreas y el escenario biogeográfico vicariante. Sin embargo, dispersión y vicarianza son procesos igualmente determinantes en la historia biogeográfica de un grupo. Gracias al uso de marcadores moleculares, es posible actualmente datar los eventos de especiación, y correlacionarlos con la aparición de la barrera paleogeográfica. En varios casos, esto ha demostrado que el grupo estudiado es demasiado joven para haberse visto afectado por la barrera vicariante, y por tanto su distribución sólo puede explicarse por dispersión (Voelker, 1999; Waters et al., 2000; Sanmartín \& Ronquist, en prensa).

Por otro lado, la idea de que la dispersión es un proceso aleatorio, al azar, y por tanto impredecible, se ha visto recientemente cuestionada por Sanmartín \& Ronquist (en prensa). Estos demuestran que si de forma repetida y continuada en el tiempo la dispersión se produce siempre entre ciertas áreas y ciertas regiones, esta puede dar lugar a patrones de distribución jerárquicos, que pueden ser reconstruidos por un cladograma de áreas, al igual que en el proceso de vicarianza. Esto se conoce como "concordant dispersal" o "dispersión predecible" (Sanmartín \& Ronquist, en prensa). En este caso, la dispersión es un fenómeno general que afecta a muchos grupos simultáneamente, por ejemplo, la dispersión entre Australia y Nueva Zelanda debida a la Corriente Antártica (Sanmartín \& Ronquist, en prensa). Si la dispersión predecible ocurre en una secuencia, el resultado es un patrón de distribución jerárquico que puede detectarse comparando la filogenia de distintos grupos de organismos de una misma región. En este último caso, la única forma de distinguir entre dispersión predecible y vicarianza es comparando el cladograma de áreas con un cladograma geológico que especifique las relaciones geológicas entre las áreas, i.e., su secuencia de fragmentación (Sanmartín \& Ronquist, en prensa).

En los últimos años se han propuesto nuevos métodos de inferencia biogeográfica en los que se consideran explícitamente los procesos de dispersión y vicarianza en la reconstrucción biogeográfica. Este tipo de métodos (denominados "event-based") reconstruyen el patrón de distribuciones ancestrales 
pero también la secuencia de procesos biogeográficos que los determinaron (Ronquist, 1997, 1998, 2002; Sanmartín \& Ronquist, 2002). A cada uno de estos procesos (dispersión, vicarianza, extinción, y duplicación) se les asigna un coste que estaría inversamente relacionado con su probabilidad: cuanto más probable el evento, menor el coste. El análisis biogeográfico consiste entonces en buscar la reconstrucción biogeográfica "más parsimoniosa", es decir, la reconstrucción que minimice el coste total de los eventos (Sanmartín \& Ronquist, 2002).

Uno de estos nuevos ("event-based") métodos de reconstrucción biogeográfica es el "análisis de dispersión- vicarianza" (Ronquist, 1997), implementado en el programa DIVA v. 1.1 (Ronquist, 1996). El análisis de dispersión-vicarianza permite reconstruir las distribuciones ancestrales de un grupo de organismos a partir de su filogenia y su distribución actual. Aunque asume un modelo vicariante como modelo nulo, se diferencia de la Biogeografía Cladística en que permite incorporar procesos como dispersión y extinción a la reconstrucción biogeográfica. Además, no restringe las relaciones entre áreas a un patrón jerárquico, el cladograma de áreas, sino que permite que las relaciones entre áreas sean reticuladas y múltiples (Ronquist, 1996). Así puede utilizarse en grupos afectados de distinta forma por sucesivos eventos geológicos (Sanmartín et al., 2001).

El objetivo de este trabajo es mostrar la utilidad del análisis de dispersión-vicarianza en la reconstrucción de la historia biogeográfica de la subfamilia Pachydeminae Reitter, 1902 (Melolonthidae, Scarabaeoidea, Coleoptera) en el Paleártico occidental, y en particular de los géneros ibéricos Elaphocera Gené, 1836 y Ceramida Baraud, 1987 en el área Mediterránea. La Cuenca Mediterránea representa un ejemplo de escenario biogeográfico "reticulado", en el que distintas placas se formaron y fragmentaron por la aparición y desaparición sucesiva de barreras dispersivas, como cuencas marinas o conexiones terrestres (Rögl \& Steininger, 1983; Oosterbroek \& Arntzen, 1992).

La subfamilia Pachydeminae constituye una herramienta ideal para estudiar el efecto de los procesos históricos en la formación de patrones biogeográficos, particularmente en el Mediterráneo. Por un lado, presenta una elevada tasa de endemicidad, probablemente debido a que las hembras son ápteras o braquípteras en la mayoría de especies, lo que ha dado lugar a rangos geográficos muy reducidos (Sanmartín \& Martín-Piera, 2003). Por otro lado, la distribución de la subfamilia a nivel mundial, aunque cosmopolita, es geográficamente muy disjunta. Por ejemplo, en la Región Neártica sólo están presentes en el extremo oeste de Norteamérica (California y Méjico), mientras que en el Paleártico, géneros como Elaphocera muestran una distribución anfimediterránea, con especies en el Mediterráneo occidental (Península Ibérica, Baleares, Cerdeña, Marruecos) y oriental (Grecia y Turquía) pero ausentes del Mediterráneo central (Francia, Italia, y África nororiental). Este tipo de disyunción mediterránea este-oeste ha sido observada en muchos otros grupos animales (Oosterbroek \& Arntzen, 1992; MartínPiera \& Sanmartín, 1999).

A pesar de que algunos géneros de Pachydeminae como Ceramida han sido descritos recientemente como plaga agrícola, este grupo ha sido relativamente poco estudiado en comparación con otras subfamilias de Melolonthidae (Baraud, 1992). Recientemente, Sanmartín \& Martín-Piera (2003) revisaron los géneros de Pachydeminae paleárticos y propusieron la primera hipótesis de relaciones filogenéticas dentro de la subfamilia. Basándose en esta hipótesis, Sanmartín (2003) reconstruyó la historia biogeográfica de la subfamilia Pachydeminae en la región paleártica (Mediterránea), utilizando el análisis de dispersión-vicarianza. Un estudio semejante está ahora en marcha (Sanmartín, in prep.) para reconstruir el origen y posibles vías de colonización de los géneros Elaphocera y Ceramida en la Península Ibérica. Un análisis preliminar se incluyó como parte de los resultados de mi Tesis Doctoral (Sanmartín, 1998). En este artículo se resumen las conclusiones de estos dos trabajos. El objetivo es mostrar la utilidad del análisis de dispersión-vicarianza a la hora de reconstruir la historia biogeográfica de un grupo de organismos en una región donde no existe un patrón biogeográfico general (relaciones entre áreas reticuladas).

\section{Material y métodos}

\section{ÁREAS DE ENDEMISMO}

La subfamilia Pachydeminae incluye 20 géneros paleárticos y alrededor de 300 especies, aunque todavía no existe un catálogo definitivo (Lacroix, 2000). Se distribuyen por el sur de Eurasia, desde las Islas Canarias hasta China, incluyendo el norte de África, sur de Europa (excepto Francia e Italia), Asia Menor, Oriente Medio, Irán y Afganistán, las repúblicas caucásicas, y Asia Central (Sanmartín \& Martín-Piera, 2003). La mayor parte de las especies se encuentran al oeste de los Urales. En el Paleártico oriental, los Pachydeminae están sólo representados por unas pocas especies en China (Lacroix, 2002). 


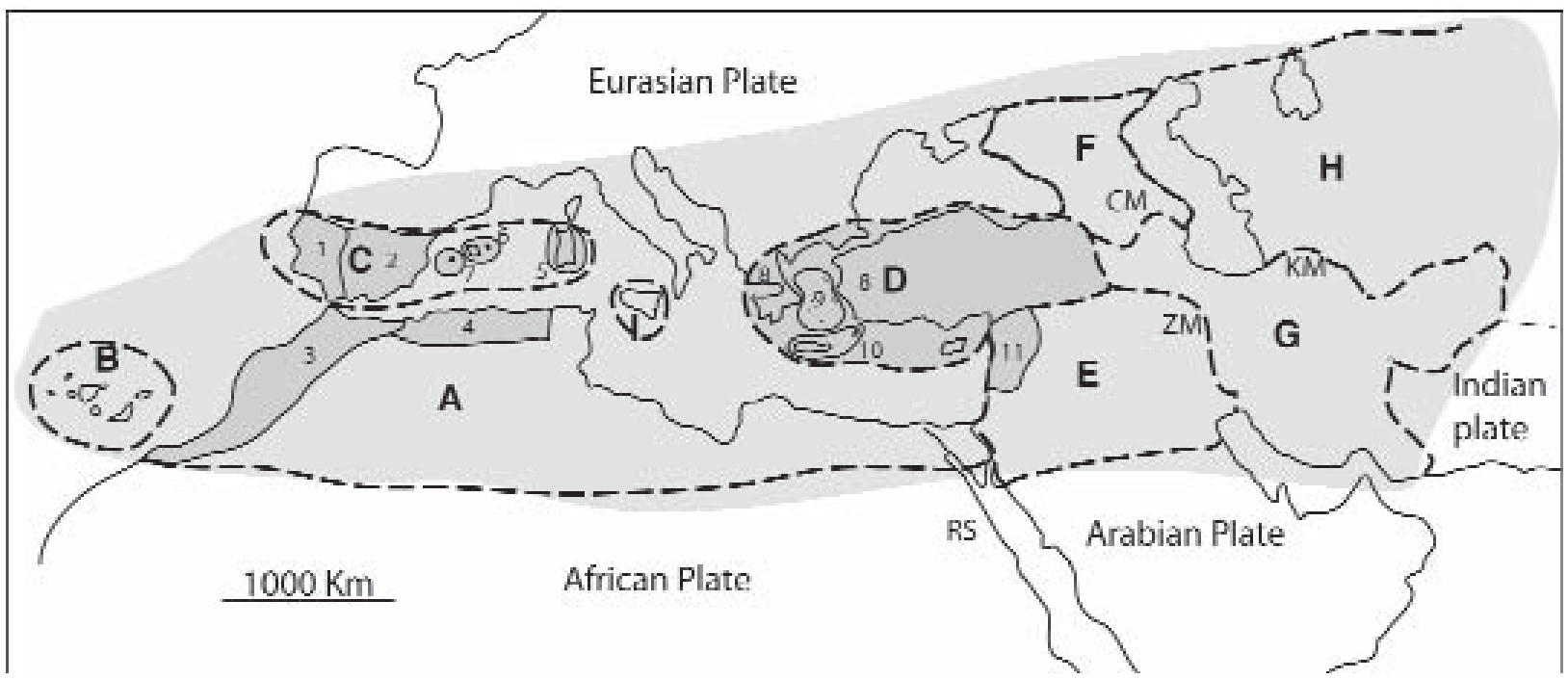

Fig. 1.- Áreas de endemismo utilizadas en la reconstrucción de la historia biogeográfica de: a) la subfamilia Pachydeminae (AI, Sanmartín, 2003), y b) los géneros ibéricos Elaphocera y Ceramida (1-11, Sanmartín, 1998). A = Norte de África, B = Islas Canarias, $\mathrm{C}=$ Mediterráneo occidental (Península Ibérica e Islas Mediterráneas suroccidentales), $\mathrm{D}=$ Mediterráneo oriental (Balcanes/Anatolia e Islas Mediterráneas suorientales), $\mathrm{E}=$ Oriente Medio, $\mathrm{F}=$ Caúcaso, $\mathrm{G}=$ Irán-Afganistán, $\mathrm{H}=\mathrm{Asia}$ Central, I = Sicilia. 1: suroeste de la Península Ibérica, 2: sureste de la Península Ibérica, 3: Magreb atlántico (Marruecos), 4: Magreb mediterráneo (Argelia), 5: Cerdeña, 6: Islas Gimnésicas (Mallorca), 7: Islas Pitiusas (Ibiza, Conejera, Formentera), 8: BalcanesAnatolia, 9: Islas del Egeo, 10) Arco insular Creta-Rodas, 11) Siria. Abreviaturas: CM: Montañas del Caúcaso, KM: Cadena montañosa del Kopet-Dagh, RS: Mar Rojo, ZM: Cadena montañosa del Zagros. Adaptado de Sanmartín (2003).

Fig. 1.- Areas of endemism used in the biogeographic analysis of: a) the subfamily Pachydeminae (A-B, Sanmartín, 2003), and b) the Iberian genera Elaphocera and Ceramida (1-11, Sanmartín, 1998). A = North Africa, B = Canary Islands, C = Western Mediterranean region (Iberian Peninsula and southwestern Mediterranean islands), D = Eastern Mediterranean (Balkans/Anatolia and southeastern Mediterranean Islands), $\mathrm{E}=$ Middle East, $\mathrm{F}=$ Caucasus region, $\mathrm{G}=$ Iran-Afghanistan, $\mathrm{H}=\mathrm{Central} \mathrm{Asia}, \mathrm{I}=$ Sicily. 1: southwestern Iberian Peninsula, 2: southeastern Iberian Peninsula, 3: northwestern Morocco, 4: northern Algeria, 5: Sardinia, 6: Northern Balearic Islands (Mallorca), 7: Southern Balearic Islands (Ibiza, Conejera, Formentera), 8: BalkansAnatolia, 9: Aegean Islands, 10) Insular Arch Crete-Rhodes, 11) Syria. Abbreviations: CM: Caucasus Mountains, KM: KopetDagh Mountains, RS: Red Sea, ZM: Zagros Mountains. After Sanmartín (2003).

Se han propuesto numerosos criterios sobre cómo definir un área de endemismo, la unidad de análisis en biogeografía histórica, sin que se haya alcanzado un consenso todavía (Morrone \& Crisci, 1995). En este trabajo, el área de endemismo se define por el criterio de simpatría (Sanmartín, 2003), es decir como una distribución congruente compartida por dos o más especies, pero al mismo tiempo limitada por discontinuidades geográficas que pudieron haber actuado como barreras dispersivas, por ejemplo, el Istmo de Suez entre África y Arabia.

De acuerdo con estos principios, Sanmartín (2003) definió nueve áreas de endemismo para el análisis biogeográfico de la subfamilia Pachydeminae (Fig. 1): A) Norte de África, B) Islas Canarias, C) Mediterráneo occidental
(Península Ibérica e Islas Mediterráneas suroccidentales (Baleares, Cerdeña), D) Mediterráneo oriental (Balcanes-Anatolia e Islas surorientales), E) Oriente Medio, F) la región del Caúcaso, G) Irán-Afganistán, H) Asia Central, y I) Sicilia.

Para el estudio de la distribución biogeográfica de Elaphocera y Ceramida, se consideraron áreas más reducidas (Fig. 1, basado en Sanmartín, 1998): 1) suroeste de la Península Ibérica, 2) sureste de la Península Ibérica, 3) Magreb atlántico (noroeste de Marruecos), 4) Magreb Mediterráneo (norte de Argelia, 5) Cerdeña, 6) Islas Gimnésicas (Mallorca), 7) Islas Pitiusas (Ibiza, Conejera, Formentera); 8) Balcanes-Anatolia, 9) Islas del Egeo, 10) Arco insular Creta-Rodas, y 11) Siria. 
Tabla 1.- Especies incluidas en el análisis filogenético de Pachydeminae en Sanmartín \& Martín-Piera (2003), y su distribución geográfica. Adaptado de Sanmartín \& Martín-Piera (2003).

Table 1.- Species included in the phylogenetic análisis of of Pachydeminae in Sanmartín \& Martín-Piera (2003), and their geographic distribution. After Sanmartín \& Martín-Piera (2003).

\begin{tabular}{|c|c|c|}
\hline Géneros & Especies & Distribución geográfica \\
\hline \multicolumn{3}{|l|}{ PACHydeminae PaLEÁRTICOS } \\
\hline \multirow[t]{2}{*}{ Otoclinius Brenske, 1896} & O. fragilis Petrovitz, 1980 & Irán \\
\hline & O. gracilipes Brenske, 1896 & Irán \\
\hline Pachydemocera Reitter, 1902 & P. lucidicollis (Kraatz, 1882) & Rodas, Siria \\
\hline \multirow[t]{2}{*}{ Leptochristina Baraud \& Branco, 1991} & L. pubimargo (Reitter, 1902) & Siria \\
\hline & L. annamariae Baraud \& Branco, 1991 & Iraq \\
\hline \multirow[t]{9}{*}{ Tanyproctus Faldermann, 1835} & T. reichei (Rambur, 1843) & Grecia; Asia Menor \\
\hline & T. rufidens (Marseul, 1879) & Irán, Caúcaso \\
\hline & T. kindermanni (Reiche, 1861) & Siria, Israel \\
\hline & T. bucharicus (Reitter, 1897) & Afganistán, Tayikistán \\
\hline & T. subciliatus Reitter, 1902 & Afganistán, Turkmenistán \\
\hline & T. saulcyi (Reiche, 1856) & Israel; Siria \\
\hline & T. rugulosus Fairmaire, 1892 & Siria \\
\hline & T. ganglbaueri (Brenske, 1897) & Irán \\
\hline & T. persicus (Ménétries, 1832) & Irán, Caúcaso \\
\hline \multirow[t]{10}{*}{ Pachydema Castelnau, 1832} & P. castanea (Brullé, 1838) & Islas Canarias \\
\hline & P. obscura (Brullé, 1838) & Islas Canarias \\
\hline & P. bipartita (Brullé, 1838) & Islas Canarias \\
\hline & P. tinerfensis Galante \& Stebnicka, 1992 & Islas Canarias \\
\hline & P. anthracina Fairmaire, 1860 & Marruecos \\
\hline & P. hirticollis (Fabricius, 1787) & Argelia; Túnez; Libia \\
\hline & P. xanthochroa Fairmaire, 1879 & Túnez \\
\hline & P. palposa Reitter, 1902 & Egipto \\
\hline & P. rubripennis (Lucas, 1848) & Marruecos; Argelia \\
\hline & P. zohra Normand, 1951 & Túnez; Libia \\
\hline \multirow[t]{6}{*}{ Hemictenius Reitter, 1897} & H. tekkensis (Reitter, 1889) & Transcaspio; Turkmenistán \\
\hline & H. ochripennis Reitter, 1902 & Tayikistán \\
\hline & H. opacus Ball, 1870 & Turkmenistán; Tayikistán \\
\hline & H. simplicitarsis Reitter, 1897 & Tayikistán \\
\hline & H. opacipes Reitter, 1902 & Buchara, Uzbekistán \\
\hline & H. nigrociliatus Reitter, 1897 & Buchara, Uzbekistán \\
\hline \multirow[t]{8}{*}{ Elaphocera Gené, 1836} & E. elongata Schauffus, 1874 & Grecia; Turquía \\
\hline & E. syriaca Kraatz, 1882 & Siria \\
\hline & E. emarginata (Gyllenhal, 1817) & Cerdeña \\
\hline & E. capdeboui Schauffus, 1882 & Islas Baleares \\
\hline & E. barbara Rambur, 1843 & Marruecos; Argelia \\
\hline & E. sulcatula Fairmaire, 1884 & Marruecos \\
\hline & E. carteiensis Rambur, 1843 & SE Península Ibérica \\
\hline & E. alonsoi López-Colón, 1992 & SE Península Ibérica \\
\hline \multirow{2}{*}{ Ceramida Baraud, 1987} & C. bedeaui (Erichson, 1840) & S. Península Ibérica; NO de África \\
\hline & C. baraudi (Branco, 1981) & Portugal \\
\hline Europtron Marseul, 1867 & E. gracile Marseul, 1867 & Argelia \\
\hline Peritryssus Reitter, 1918 & P. excisus Reitter, 1918 & Sicilia \\
\hline Brenskiella Berg, 1898 & B. flavomicans (Brenske, 1896) & Israel \\
\hline Alaia Petrovitz, 1980 & A. sexdentata Petrovitz, 1980 & Irán; Afganistán \\
\hline Tanyproctoides Petrovitz, 1971 & T. arabicus (Arrow, 1932) & Arabia Saudi \\
\hline \multirow[t]{2}{*}{ Atanyproctus Petrovitz, 1954} & A. miksici Petrovitz, 1965 & Afganistán \\
\hline & A. simplicitarsis Petrovitz, 1954 & Irán \\
\hline Pseudopachydema Balthasar, 1930 & P. caucasica Balthasar, 1930 & Caúcaso \\
\hline Kryzhanovskia Nikolajev \& Kabakov, 1977 & K. olegi Nikolajev \& Kabakov, 1977 & Afganistán \\
\hline \multicolumn{3}{|l|}{ PACHYDEMINAE NO-PALEÁRTICOS } \\
\hline Sparrmannia La Porte, 1840 & S. alopex (Fabricius, 1787) & Sudáfrica \\
\hline Phobetus Le Conte, 1856 & P. comatus Le Conte, 1856 & California \\
\hline \multicolumn{3}{|l|}{ GRUPO EXTERNO } \\
\hline Euserica Reitter, 1896 & E. mutata (Gyllenhal, 1817) & Península Ibérica, Marruecos \\
\hline Melolontha Fabricius, 1775 & M. papposa Illiger, 1803 & Península Ibérica \\
\hline Pseudotrematodes Jaquelin du Val, 1860 & P. friwaldskii (Menétriès, 1836) & Balcanes, Asia Menor \\
\hline Polyphylla Harris, 1842 & P. fullo (Linnaeus, 1758) & $\begin{array}{l}\text { Europa central y occidental, Rusia, } \\
\text { Caúcaso, N África, Asia Menor }\end{array}$ \\
\hline Aplidia Hope, 1837 & A. akbesiana Petrovitz, 1971 & Siria \\
\hline
\end{tabular}




\section{HiPÓTESIS FILOGENÉTICA}

Sanmartín \& Martín-Piera (2003) incluyeron en su análisis 16 de los 20 géneros paleárticos, representados por 49 especies. Los cuatro géneros paleárticos restantes son monoespecíficos o de posición dudosa (Sanmartín \& Martín-Piera, 2003). En la elección de las especies se intentó representar la variabilidad geográfica y morfológica de cada género. La Tabla 1 muestra las especies incluidas en el análisis y su distribución geográfica (Sanmartín \& Martín-Piera, 2003). Las relaciones filogenéticas entre las distintas tribus o subfamilias de Melolonthidae no han sido todavía bien estudiadas (Browne \& Scholtz, 1999). Sin embargo, Melolonthinae se ha considerado tradicionalmente como el grupo hermano o muy cercano filogenéticamente a Pachydeminae (Baraud, 1992), y los géneros paleárticos han sido recientemente objeto de una revisión sistemática (Coca-Abia, 1995). Por tanto, Sanmartín \& Martín-Piera (2003) utilizaron Melolonthinae como grupo externo en la filogenia, incluyendo dos géneros por cada una de las tribus Rhizotrogini y Melolonthini (Coca-Abia, 1995). Incluyeron también un representante de la subfamilia Sericinae (Euserica Reitter, 1896). Asimismo, puesto que ningún estudio filogenético previo había corroborado que los Pachydeminae paleárticos integran un grupo monofilético dentro de la subfamilia, Sanmartín \& Martín-Piera (2003) incluyeron en el análisis dos géneros de Pachydeminae no paleárticos: el género surafricano Sparrmannia La Porte, 1840 y el neártico Phobetus Le Conte, 1856.

La Figura 2A muestra el árbol de consenso estricto de los 32 árboles más parsimoniosos obtenidos en el análisis filogenético de la subfamilia (Sanmartín \& Martín-Piera, 2003). En esta filogenia, los Pachydeminae paleárticos aparecen como un grupo monofilético con respecto a los representantes de Melolonthinae y Sericinae, pero también con respecto a los géneros de Pachydeminae no paleárticos. Esto último, sin embargo, necesita ser corroborado con un estudio más detallado, ya que tanto Sparmannia como Phobetus no pudieron ser codificados para los caracteres genitales femeninos (Sanmartín \& Martín-Piera, 2003). Asimismo, la filogenia de estos autores no incluyó ninguna de las especies chinas de Pachydeminae. La distribución de estas especies es muy disjunta con respecto al resto de la subfamilia, y se ha considerado a veces como parte de la región oriental. Sería interesante, hacer un nuevo análisis incluyendo estas especies para examinar si derivaron de uno de los linajes paleárticos, o forman un grupo independiente (Sanmartín, 2003). En el análisis de los géneros ibéricos Elaphocera y Ceramida se utilizó al géne- ro Leptochristina Baraud \& Branco, 1991 como grupo externo, siguiendo la topología de la filogenia de la subfamilia (Figura 2A). Se obtuvieron cuatro árboles (MPTs), cuyo consenso estricto se muestra en la Figura 2B (Sanmartín, 1998).

Uno de los requisitos del análisis de dispersiónvicarianza es que la filogenia debe estar completamente resuelta, es decir, no pueden existir politomías en el árbol. Puesto que el número de árboles originales en el caso de la subfamilia es demasiado grande (32 MPTs), se utilizó uno de los árboles más parsimoniosos como hipótesis filogenética de partida (Figuras 3 y 4), aunque las conclusiones biogeográficas discutidas abajo se basan sólo en los clados recogidos en el árbol consenso, es decir corroboradas por todos los árboles originales (Sanmartín, 2003).

\section{ANÁLISIS BIOGEOGRÁFICO}

En la reconstrucción histórica se empleó el análisis de dispersión-vicarianza (Ronquist, 1997) implementado en el programa DIVA v. 1.1 (Ronquist, 1996). DIVA reconstruye las distribuciones ancestrales en cada nodo de la filogenia de acuerdo con un simple modelo de especiación alopátrica por vicarianza, pero al mismo tiempo permite considerar otros procesos como dispersión y extinción como posibles explicaciones de la distribución geográfica.

El análisis considera cuatro tipos de procesos biogeográficos a los que asigna un coste distinto: a) vicarianza (especiación alopátrica en respuesta a una barrera general que afecta a muchos grupos simultáneamente): el coste de este evento es cero ya que este es el "modelo nulo" en análisis biogeográfico; b) duplicación (especiación dentro del área, equivalente a especiación simpátrica, o más a menudo a especiación alopátrica en respuesta a una barrera temporal local): el coste de este evento es cero porque no altera la distribución ancestral, es decir, equivaldría a una ausencia de "cambio de estado" en inferencia filogenética; c) dispersión (a través de una barrera preexistente seguida de especiación alopátrica): recibe un coste de uno por cada una de las áreas añadidas a la distribución del taxón. d) extinción (desaparición de un taxón o linaje de una de las áreas donde se predice su presencia): recibe un coste de uno por cada área eliminada de la distribución ancestral.

Al igual que otros métodos de reconstrucción biogeográfica, el "modelo nulo" en DIVA está basado en la especiación alopátrica por vicarianza geográfica (aunque considera que la dispersión juega un papel importante en la especiación). Esto es así porque, teóricamente, la especiación alopátrica es el tipo de especiación más frecuente en organismos terrestres (Ronquist, 1997). Además, 
A)

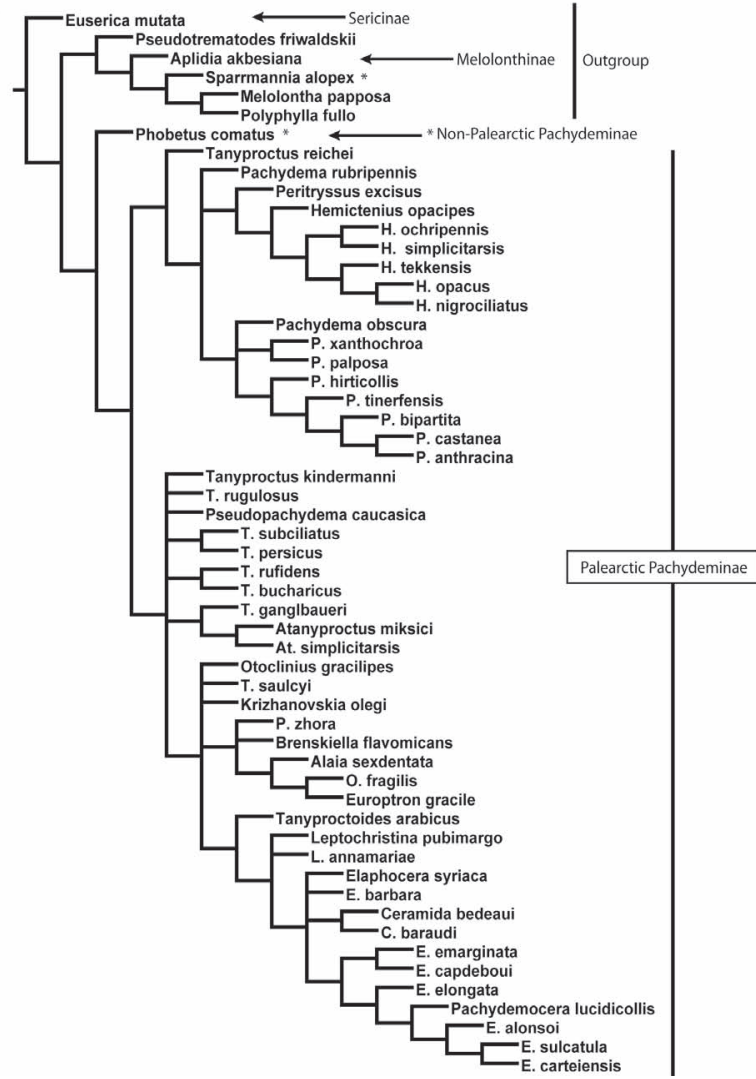

B)

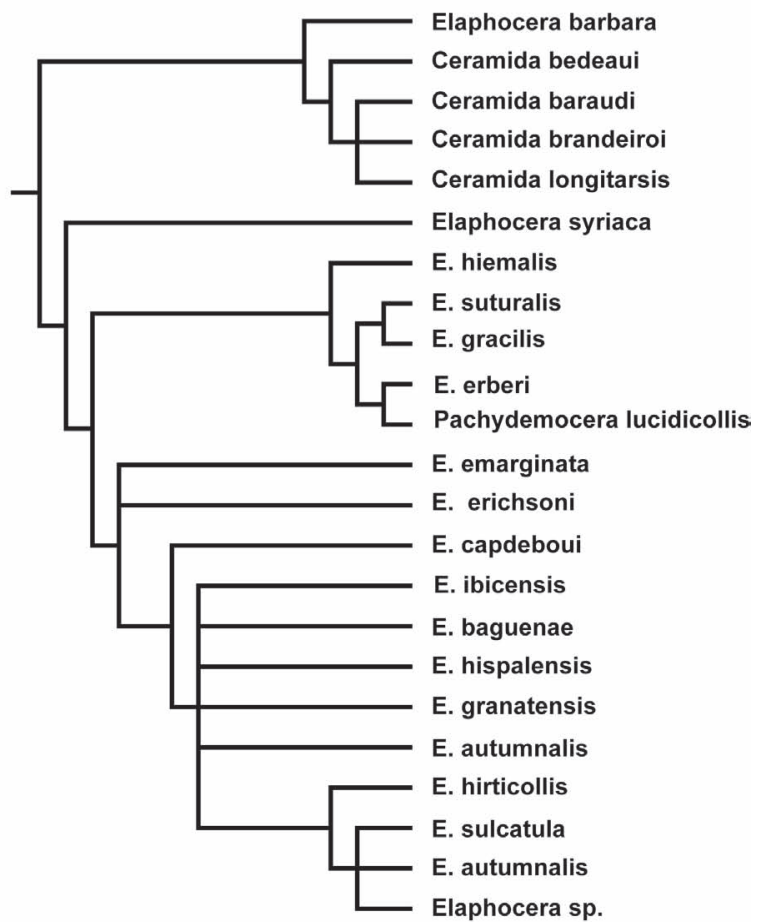

Fig. 2.- A) Filogenia de los Pachydeminae Paleárticos (Sanmartín \& Martín-Piera, 2003); consenso estricto de los 32 árboles más parsimoniosos (MPTs) de longitud 284 pasos $(\mathrm{CI}=0.35 ; \mathrm{RI}=0.77)$. B) Filogenia de los géneros ibéricos de Pachydeminae Elaphocera y Ceramida (Sanmartín, 1998); consenso estricto de cuatro árboles más parsimoniosos $(\mathrm{L}=175, \mathrm{CI}=0.62, \mathrm{RI}=0.83$ ).

Fig. 2.-A) Phylogeny of the Palearctic Pachydeminae (Sanmartín \& Martín-Piera, 2003); strict consensus of 32 most parsimonious trees (MPTs) of 284 steps $(\mathrm{CI}=0.35 ; \mathrm{RI}=0.77)$. B) Phylogeny of the Iberian genera of Pachydeminae Elaphocera and Ceramida (Sanmartín, 1998); strict consensus of four MPTs of 175 steps $(\mathrm{CI}=0.62, \mathrm{RI}=0.83$ ).

tanto dispersión como extinción son eventos no predecibles que pueden borrar las trazas biogeográficas de procesos que están "filogenéticamente conservados" y que por tanto son predecibles, como por ejemplo, vicarianza o expansión geográfica entre áreas que han vuelto a unirse (difusión) (Sanmartín \& Ronquist, 2002). En el proceso de vicarianza, cada división geográfica es seguida de especiación alopátrica por lo que los descendientes "heredan" su distribución geográfica de la especie ancestral; lo mismo sucede en la duplicación. En la dispersión, en cambio, el área se adquiere de forma independiente, y por tanto no puede predecirse "filogenéticamente". Si procesos aleatorios como dispersión o extinción no son penados en la reconstrucción biogeográfica asignándoles un coste, se corre el riesgo de introducir elementos espurios en las reconstrucciones óptimas (Ronquist, 1997, 1998; Sanmartín \& Ronquist, 2002). La reconstrucción biogeográfica óptima será aquella que minimice el número de dispersiones y extinciones. DIVA por tanto utiliza un criterio de máxima parsimonia como criterio de optimización en inferencia biogeográfica.

Otra razón para "penalizar" dispersión y extinción en la reconstrucción biogeográfica es que estos procesos afectan a cada taxón independientemente del área que ocupa, e independientemente de otros organismos (excepto en "dispersión predecible", ver Sanmartín \& Ronquist, en prensa), mientras que la vicarianza considera conjuntamente la evolución de áreas y organismos (lo que constituye el objetivo 
mismo de la biogeografía histórica) y afecta a muchos organismos simultáneamente (la duplicación no afecta a la reconstrucción de áreas ancestrales porque implica que no existe un cambio de área).

No obstante, un inconveniente con el análisis de dispersión-vicarianza es que la reconstrucción de las distribuciones ancestrales se hace más ambigua a medida que se acerca al nodo raíz del árbol. Esto se debe a que la optimización en cada nodo es tridimensional y tiene en cuenta la información de los dos descendientes y del ancestro, mientras que en el caso del nodo raíz, esta última no existe. Como resultado, la distribución ancestral de la raíz suele incluir todas o casi todas las áreas analizadas. Para evitar esto, se puede incluir en el análisis uno o varios grupos externos que limiten la posible distribución ancestral. En el caso de Pachydeminae, sin embargo, los taxones que forman el grupo externo (Sanmartín \& MartínPiera, 2003) se distribuyen fuera del área estudiada (e. g., Phobetus) o son de amplia distribución (Melolonthinae, ver Tabla 1). Otra posibilidad es realizar un análisis "restringido" (Ronquist, 1996), en el que se limitan el número de áreas permitidas en la distribución ancestral del grupo. Esto equivale a plantearse: si el ancestro del grupo no estaba presente en todas las áreas que ahora ocupa sino que se originó en un "centro de origen" (una o varias áreas) desde donde se dispersó al resto de las áreas, cuáles serían las áreas más probables de formar parte de esta distribución ancestral? (Sanmartín, 2003). Una forma de restringir el número de áreas es considerar el número máximo de áreas en el que se distribuye cualquiera de los representantes actuales del grupo, y restringir las distribuciones ancestrales de acuerdo con este criterio. En el caso del análisis de la subfamilia en el Paleártico, resulta poco probable que el ancestro de los Pachydeminae Paleárticos estuviera presente en una distribución tan extensa que abarcara todas las áreas analizadas (el sureste de Europa y Asia), por lo que se restringió el número de áreas a cuatro (ver Sanmartín, 2003 para una explicación más detallada). En el caso de los géneros ibéricos Elaphocera y Ceramida, se incluyó a Leptochristina como grupo externo en el análisis biogeográfico; este género se distribuye principalmente en el Oriente Medio.

\section{Resultados y discusión}

Evolución BIOGEOGRÁFICA DE LA SUBFAMILIA Pachydeminae en el PALEÁRTico occidental

La Figura 3 reproduce la reconstrucción de la historia biogeográfica de la subfamilia Pachydeminae en el Paleártico occidental, según el análisis de dispersión-vicarianza en Sanmartín (2003). A continuación se resumen las principales conclusiones de este trabajo.

1) Las reconstruccion óptima postulada por DIVA indica que los Pachydeminae paleárticos probablemente se originaron en la región Mediterránea sur-oriental, incluyendo el Norte de África, Oriente Medio, la Meseta Iraní (IránAfganistán), y las Penínsulas Balcánica y Anatólica. Por tanto, la presencia de los Pachydeminae en áreas como el Mediterráneo occidental, el Archipiélago Canario, el Caúcaso, o Asia Central, sería el resultado de una dispersión posterior desde el área ancestral.

2) Durante el Oligoceno-Mioceno, a mediados del Terciario, la colisión de las Placas Africana, Arábiga, y Eurasiática dio lugar a la aparición de sucesivas barreras dispersivas que dividieron la distribución ancestral, y dieron origen a varios géneros en sucesivos eventos de vicarianza. La primera vicarianza aisló al ancestro del clado Pachydema Castelnau, 1832 - Hemictenius Reitter, 1897 -Tanyproctus reichei (Rambur, 1843) en el Norte de África y la región de los Balcanes-Anatolia del resto de los Pachydeminae paleárticos en el Oriente Medio y la Meseta Iraní (AD/EG). Sanmartín (2003) explica esta vicarianza como resultado de la apertura del Mar Rojo y el Istmo de Suez en el Terciario. A finales del Cretácico y comienzos del Terciario, hace 60 millones de años (Ma), la Placa Arábiga comenzó a separarse de la gran Placa Africana a lo largo de la línea del Mar Rojo, que marca la línea geográfica entre estas dos placas (Thompson, 2000). El proceso de separación comenzó en el sur pero continuó durante todo el Terciario, hasta que, a comienzos del Plioceno (5 Ma), el mar Rojo se abrió finalmente al Mediterráneo a través del Istmo de Suez. Esto supuso una interrupción de las conexiones terrestres entre el Norte de África (la Placa Africana) y el Oriente Medio o Asia occidental (la Placa Arábiga), con el consiguiente aislamiento de las biotas a ambos lados del Mar Rojo. Otros autores coinciden en señalar que la apertura del Mar Rojo, y la vicarianza resultante, son probablemente la principal causa de las importantes divergencias evolutivas encontradas entre linajes norteafricanos y asiático-occidentales (Doadrio, 1990; Cheylan, 1995; Doadrio et al., 1998).

3) El segundo evento de vicarianza separó a Tanyproctus reichei en el Mediterráneo Oriental 


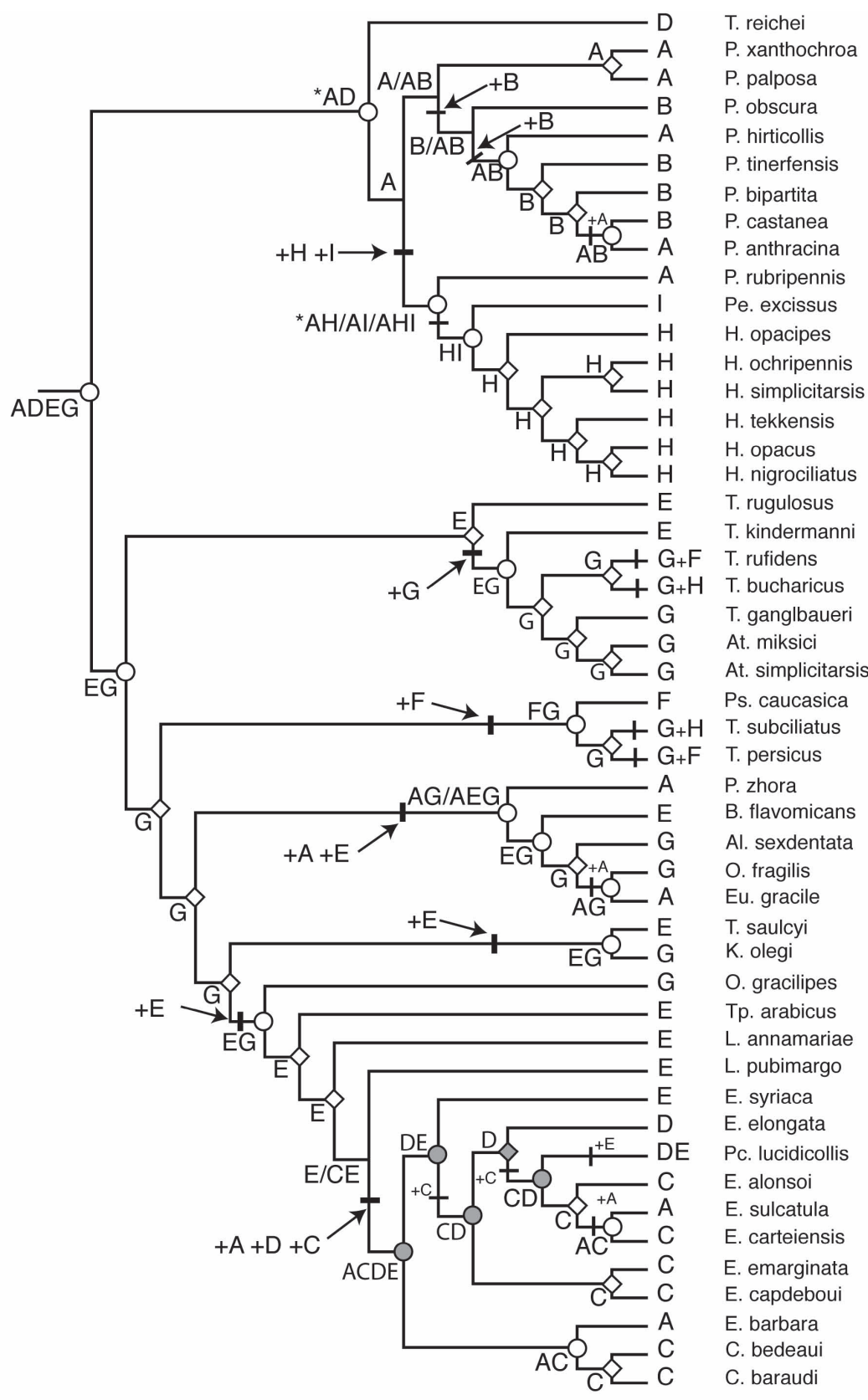

Fig. 3.- Reconstruccion de la historia biogeográfica de los Pachydeminae Paleárticos, según el análisis de dispersión-vicarianza. Adaptado de Sanmartín (2003). La filogenia representa uno de los 32 MPTs en Sanmartín \& Martín-Piera (2003). En cada uno de los nodos se han representado las distribuciones ancestrales óptimas, con las distribuciones alternativas separadas por una barra. En el caso de Elaphocera y Ceramida existen múltiples alternativas y sólo una de las posibles reconstrucciones se señala en la figura. La reconstrucción requiere 23 eventos de dispersión. Símbolos: círculo (vicarianza), rombo (duplicación), flecha (dispersión), asterisco (extinción en las áreas intermedias). Abreviaturas de áreas (ver Figura 1).

Fig. 3.- Reconstruction of the biogeographic history of the Palearctic Pachydeminae according to dispersal-vicariance analysis. After Sanmartín (2003). The phylogeny is one of the 32 MPTs in Sanmartín \& Martín-Piera (2003). At each node, the optimal distribution is given; alternative, equally optimal distributions are separated with slash. In the case of Elaphocera and Ceramida, there were multiple possible reconstructions, and only one is given in the figure. The optimal reconstruction requires 23 dispersal events. Symbols: circle (vicariance), rhombus (duplication), arrow (dispersal), asterisk (extinction event in the intermediate areas). See Figure 1 for area abbreviations. 
del ancestro de Pachydema y Hemictenius en el Norte de África (A/D). Durante el Mioceno medio (14-13 Ma), los intercambios faunísticos entre Europa y África se hicieron posibles a través de un puente continental que separaba el Tethys (protoMediterráneo) del Paratethys, y que se correspondía con la región de los Balcanes-Anatolia. Esta conexión se interrumpió en el Mioceno superior (10 Ma), cuando una nueva transgresión marina en el este, aisló el bloque balcánico-anatólico del resto del Mediterráneo (Rögl \& Steininger, 1983; Oosterbroek \& Arntzen, 1992). Sanmartín (2003) señala que $T$. reichei es probablemente el único superviviente de un linaje que estaba originalmente presente en el Mediterráneo oriental (D) pero quedó posteriormente aislado a consecuencia de la transgresión marina que restauró las conexiones entre el Tethys y el Paratethys a finales del Mioceno. La posibilidad alternativa sería una dispersión reciente desde el Norte de África a Asia Menor, pero el carácter relicto de $T$. reichei, con numerosas apomorfías, sugiere la primera explicación como la más probable (Sanmartín, 2003). Más difícil es explicar cómo el clado PachydemaHemictenius-T. reichei adquirió, previa a la vicarianza, una distribución geográfica tan disjunta (AD). Sanmartín (2003) sugiere que probablemente en un principio la distribución del ancestro incluía también la región del Levante, situada entre el Norte de África y los Balcanes-Asia Menor, pero que luego se extinguió en esta área (ver Fig. 3).

4) Finalmente, el tercer evento de vicarianza separó al ancestro de Atanyproctus Petrovitz, 1954 y un grupo de especies de Tanyproctus Faldermann, 1835 (este género es polifilético según Sanmartín \& Martín-Piera, 2003) en el Oriente Medio, del ancestro del resto de Pachydeminae en Irán-Afganistán (E/G). Sanmartín (2003) explica esta separación como resultado de la elevación de la cadena montañosa del Zagros en el sur de Irán, como consecuencia de la colisión de la Placa Arábiga con la Placa Eurasiática a finales del Mioceno (10 Ma). Una segunda fase orogénica a comienzos del Plioceno (5 Ma) habría dado lugar a las montañas del Caúcaso y Kopet-Dagh en el norte de Irán, que marcan las fronteras geográficas con las regiones del Caúcaso y Asia Central, respectivamente. Esta secuencia orogénica ha sido sugerida para explicar divergencias evolutivas entre linajes iraníes y arábicos, y entre linajes iraníes y asiáticos, en otros grupos animales paleárticos (Cheylan, 1995; Macey et al., 1998; Martín-Piera \& Sanmartín, 1999).

5) El análisis biogeográfico (Fig. 3) también postula que los géneros Pachydema, Hemictenius, y Peritryssus Reitter, 1918 se originaron a partir de un ancestro norteafricano (A) que dio lugar a dos linajes por duplicación (especiación dentro del área): el ancestro del género Pachydema, que posteriormente se habría dispersado a las Islas Canarias (+B), y el ancestro del clado Pachydema rubripennis (Lucas, 1848)Peritryssus-Hemictenius, que luego se dispersó a Sicilia $(+\mathrm{I})$ y Asia Central $(+\mathrm{H})$. El hecho de que las especies canarias de Pachydema no constituyan un grupo monofilético, sino que algunas estén más cercanas filogenéticamente a otras especies africanas de Pachydema (Sanmartín \& Martín-Piera, 2003), sugiere que la colonización del Archipiélago se produjo de forma independiente a partir de distintos ancestros africanos (Sanmartín, 2003). El ancestro del clado Peritryssus-Hemictenius se dispersó posteriormente a Sicilia $(+\mathrm{I})$, dando origen a Peritryssus excisus Reitter, 1918, y hacia el Asia Central $(+\mathrm{H})$, donde el ancestro de género Hemictenius se diversificó rápidamente. Sanmartín (2003) señala que probablemente la elevación de las cadenas montañosas del Caúcaso y Kopet-Dagh en el norte de Irán (5 Ma) contribuyó también al aislamiento del género Hemictenius en Asia Central.

6) En el resto del árbol consenso, el elevado número de politomías dificulta la reconstrucción biogeográfica. Sin embargo, se pueden extraer varias conclusiones (Sanmartín, 2003): a) El Oriente Medio (E) y la Meseta Iraní (G) habrían actuado como centros de diversificación durante la evolución de los Pachydeminae paleárticos ya que muchos de los géneros con menor número de especies de Pachydeminae se originaron en estas regiones por duplicación o especiación simpátrica (e.g., Otoclinius Brenske, 1896, Leptochristina). b) Durante la evolución biogeográfica de estos géneros, se produjeron numerosas dispersiones entre estas dos regiones y la región del Caúcaso $(+F)$. De hecho, esta última no alberga endemismos (excepto Pseudopachydema caucasica Balthasar, 1930), sino que aparece como parte de una distribución más amplia que incluye Irán o el Oriente Medio, lo que sugiere que la región del Caúcaso habría sido colonizada recientemente desde estas dos regiones (Sanmartín, 2003). 3) El 


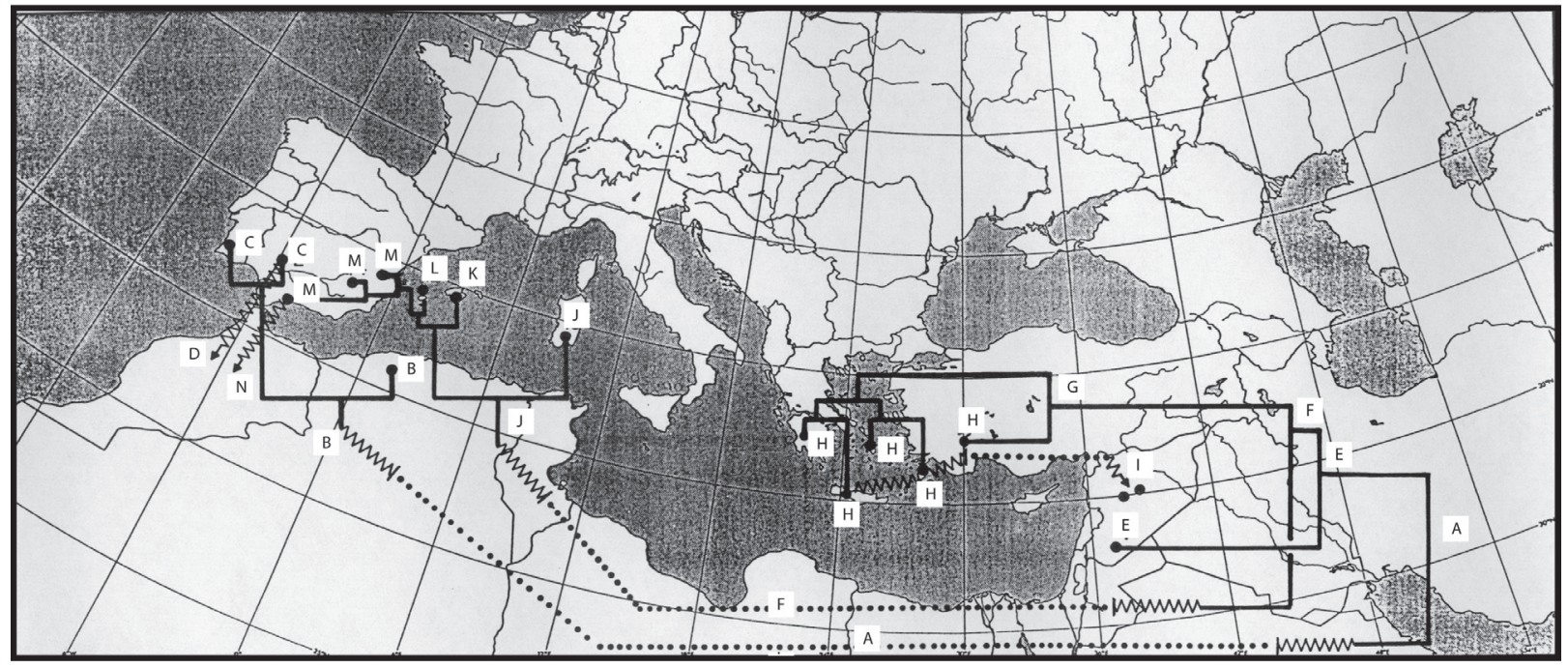

Fig. 4.- Mapa mostrando las probables rutas de dispersión y colonización de los Pachyhydeminae ibéricos Elaphocera y Ceramida en el área Mediterránea y la Península Ibérica, inferidas mediante el análisis de dispersión-vicarianza. Las letras en el cladograma indican los eventos biogeográficos discutidos en el texto. Adaptado de Sanmartín (1998).

Fig. 4.- Map showing the probable routes of dispersal and colonization of the Mediterranean and Iberian Peninsula by the Iberian Pachydeminae Elaphocera and Ceramida, inferred by dispersal-vicariance analysis. The letters in the cladogram indicate biogeographic events discussed in the text. After Sanmartín (1998).

ancestro de los géneros ibéricos Ceramida y Elaphocera probablemente se originó en el Oriente Medio y desde allí se dispersó a la región Mediterránea occidental, donde tuvo lugar la primera vicarianza entre Elaphocera y Ceramida. Este último aspecto se analiza a continuación.

EVOLUCIÓN BIOGEOGRÁFICA DE LOS GÉNEROS IBÉRICOS ELAPHOCERA Y CERAMIDA

La Figura 4 representa en forma de cladograma un resumen de la historia biogeográfica de los géneros ibéricos Elaphocera y Ceramida en el Mediterráneo y la Península Ibérica basado en el análisis de dispersión-vicarianza en Sanmartín (1998). A continuación se resumen las principales conclusiones. Las letras indican los eventos representados en el cladograma.

1) Como se menciona anteriormente, DIVA postula que el ancestro de Ceramida y Elaphocera se dispersó desde un área ancestral que comprendía el Oriente Medio hacia el Mediterráneo occidental: la Península Ibérica y el noroeste de África. Esta dispersión fue seguida de una vicarianza (A) que aisló al ancestro de Ceramida-Elaphocera barbara Rambur, 1843, en el noroeste de ÁfricaPenínsula Ibérica, del ancestro del género
Elaphocera en el Oriente Medio. Esta dispersión hacia el oeste pudo haber seguido dos rutas distintas. Una de ellas implica que el ancestro se dispersó por el norte a través de Europa. Durante el Mioceno, existieron varias conexiones a través de puentes terrestres entre el Mediterráneo occidental y el oriental, que facilitaron la dispersión de linajes transmediterráneos (Oosterbroek \& Arntzen, 1992). Sin embargo, esta ruta europea habría estado dificultada por la existencia de barreras montañosas como los Balcanes, los Alpes, o la cadena de los Pirineos. La segunda ruta posible de dispersión sería por el sur a través de África. Esta ruta parece la más probable. Por un lado, la distribución de las especies de Elaphocera y Ceramida en la Península Ibérica se restringe a la mitad sur lo que indica que la Meseta habría actuado como una importante barrera montañosa en la dispersión de estos géneros. Por otro, durante la "crisis de salinidad" del Mesiniense a finales del Mioceno (7-6 Ma), el Mar Rojo se habría secado parcialmente en el norte, permitiendo la dispersión de linajes desde el Oriente Medio hacia el Norte de África. Posteriormente, la desecación parcial que sufrió el Mediterráneo durante la crisis del Mesiniense 
habría permitido la dispersión del ancestro de Ceramida desde el Norte de África hacia la Península Ibérica a través del Estrecho de Gibraltar. Una invasión de la Península Ibérica por ancestros asiáticos (Oriente Medio-Asia Menor) a través del Norte de África ha sido postulada en otros grupos animales (Doadrio, 1990; Doadrio et al., 1998; Martín-Piera \& Zunino, 1985). Finalmente, el taxón mas basal del clado que dio origen a Ceramida, Elaphocera barbara, es endémico del Norte de África, lo que apoyaría la idea de que el ancestro de Ceramida se dispersó a la Península Ibérica desde África, y no a través de los Pirineos.

2) Posteriormente, un segundo evento de vicarianza (B) habría separado a Elaphocera barbara en el Norte de África (Marruecos) del ancestro del género Ceramida, en el suroeste de la Península Ibérica. Esta división pudo deberse a la apertura del Estrecho de Gibraltar a finales del MiocenoPlioceno (5 Ma), cuando el Mediterráneo se abrió de nuevo al Atlántico al terminar la crisis de salinidad del Mesiniense. En la Península, el ancestro de Ceramida se habría diversificado rápidamente $(\mathrm{C})$, y luego dispersado de nuevo a la costa africana, Marruecos, a través del estrecho de Gibraltar (D).

3) La primera vicarianza dentro del género Elaphocera (E) separó a Elaphocera syriaca Kraatz, 1882 en el área ancestral (Oriente Medio: Siria) del ancestro del resto de las especies. Este se habría dispersado posteriormente hacia el Mediterráneo oriental (región de los Balcanes-Anatolia) y hacia el oeste (Mediterráneo occidental). Esta dispersión dio lugar a un segundo evento de vicarianza EsteOeste (F) entre el ancestro de las especies de Elaphocera en el Mediterráneo occidental (Península Ibérica, Baleares y Cerdeña) y el stock ancestral de las especies del Mediterráneo oriental (Balcanes-Anatolia e Islas de Mediterráneo oriental).

4) En el Mediterráneo oriental, las reconstrucciones biogeográficas en DIVA indican sucesivos eventos de dispersión y vicarianza $(\mathrm{G}-\mathrm{H})$ entre la Península Balcánica (Grecia), la Península Anatólica (Asia Menor), y las Islas griegas del Egeo. Estos eventos habrían dado lugar al grupo de especies orientales de Elaphocera (e. g., E. hiemalis Erichson, 1840, E. erberi Kraatz, 1882, E. suturalis Schauffus, 1874) y Pachydemocera lucidicollis (Kraatz, 1882) (sinonimizada con
Elaphocera lucidicollis Kraatz, 1882 por Sanmartín \& Martín-Piera, 2003). Algunas de estas especies (I) se habrían dispersado posteriormente al Oriente Medio (Siria). Las dispersiones continentales entre la Península Helénica, anatólica, y Siria (H-I) pudieron producirse durante la crisis del Mesiniense o durante las regresiones marinas del Pleistoceno, y habrían utilizado a las Islas del Egeo y al Arco Creta-Rodas como puentes de conexión. Muchas de las especies presentes en Siria o Anatolia se encuentran también en Creta, Rodas, o en las Islas del Egeo (E. suturalis, P. lucidicollis, E. gracilis Waltl, 1838).

5) En el Mediterráneo occidental, la reconstrucción indica que el ancestro de las especies occidentales de Elaphocera dio lugar a las especies endémicas de la Península Ibérica, Baleares y Cerdeña en sucesivas vicarianzas (J-M). La secuencia de vicarianzas parece haber seguido una dirección Este - Oeste. Las primeras especies en quedar aisladas, i.e., las más basales en el clado occidental de Elaphocera, corresponden a Elaphocera erichsoni Jaquelin du Val, 1860, y E. emarginata (Gyllenhal, 1817), ambas endémicas de la Isla de Cerdeña (J). Le siguen una vicarianza que aisló primero a Elaphocera capdeboui Schauffus, 1882, en las Islas Baleares más orientales (Mallorca, Islas Gimnésicas, (K)), y a Elaphocera ibicensis Escalera, 1926, en las Islas Baleares más occidentales (Ibiza, Islas Pitiusas, (L)). Posteriormente, nuevas vicarianzas (M) dieron lugar al grupo de especies ahora endémicas de la mitad sureste de la Península Ibérica (e. g., E. baguenae Mancini, 1925, E. hispalensis Rambur, 1843). Finalmente, el ancestro de $E$. sulcatula Fairmaire, 1884 se habría dispersado desde la Península Ibérica al Norte de Africa (N), donde es ahora endémica.

Varias hipótesis pueden esgrimirse para explicar esta sucesión de eventos de vicarianza. Una posibilidad es que estas vicarianzas (y la dispersión que las precedió) se hubiera producido durante las regresiones marinas del Pleistoceno. Estas habrían permitido a muchos organismos dispersarse a través del Mediterráneo, para luego quedar aislados durante las transgresiones marinas posteriores (Coca-Abia, 1995). No obstante, el estudio de fósiles cuaternarios ha mostrado que la tasa de especiación en insectos fue probablemente muy baja durante el Pleistoceno (Coope, 1970). En Elaphocera, es difícil sostener que una colonización tan reciente como la del Pleistoceno hubiera dado lugar a especies insulares tan diferenciadas morfológicamente de las continen- 
tales como E. erichsoni o E. emarginata. Otra posibilidad es que la dispersión se produjera a través de los puentes terrestres que se formaron durante la crisis de salinidad del Mesiniense hace 7-6 Ma. La Greca (1990), sin embargo, sostiene que la salinidad de los suelos y las condiciones de aridez extrema que imperaron en esa época no favorecieron la dispersión terrestre de insectos. Esto es especialmente importante en el caso de Pachydeminae, cuyas especies están ligadas durante la mayor parte de su ciclo biológico (el estado larvario) a la Rizosfera. Finalmente, esta secuencia de fenómenos de vicarianza podría explicarse por eventos mucho más antiguos, como la separación del Arco Córcega-Cerdeña del Arco Bético-Rifeño en el Mioceno (De Jong, 1998). Ambas áreas se formaron como parte de la orogenia Alpina del Oligoceno superior, como resultado de la colisión de la microplaca Africana (Apulia) con la Placa Eurasiática (De Jong, 1998).

\section{VICARIANZA IBÉRICA CERAMIDA/ELAPHOCERA}

Con la excepción de Elaphocera cacerensis (López-Colón \& Rodríguez-Arias, 1986), probablemente el resultado de una dispersión posterior, el género Elaphocera se restringe a la mitad sur-oriental de la Península Ibérica, mientras que las especies del género Ceramida se distribuyen en la mitad sur-occidental de la Península. Esta vicarianza "Este-Oeste" dentro de la Península Ibérica (Íbero Atlántica/Mediterránea) se ha encontrado también en otros géneros de Melolonthidae (e. g., Rhizotrogus Berthold, 1827, Coca-Abia, 1995) y parece deberse a factores histórico-ecológicos. La Iberia Atlántica se caracteriza por suelos ácidos, de origen granítico, y relativamente húmedos, además de por un clima más pluvioso, y por una vegetación de tipo atlántico. $\mathrm{La}$ Iberia Mediterránea presenta suelos calizos, de origen sedimentario, con menor humedad, y un clima de pluviosidad más reducida, mayor relieve geográfico y una vegetación autóctona de tipo mediterráneo. Se ha demostrado que la distribución de las especies en muchos grupos de Melolonthidae está condicionada por factores físico-químico-biológicos relacionados con el suelo (Villalobos, 1991; Villalobos \& Lavelle, 1990). En el caso de Pachydeminae, esto se ve reforzado por el hecho de que el insecto pasa la mayor parte de su período vital en estado larvario, enterrado bajo el suelo. Por tanto, los factores ecológicos relacionados con el ambiente edáfico (humedad y textura del suelo, biomasa radicícola) son determinantes en la distribución de las especies. En el género Ceramida se ha podido comprobar que la humedad del suelo es un factor determinante en la mortalidad larvaria (Sanmartín, submitted).

\section{AGRADECIMIENTOS}

Agradezco a Jorge M. Lobo su invitación a participar en este homenaje de Graellsia al trabajo de Fermín Martín Piera. Este artículo se basa en los resultados de mi Tesis Doctoral (Universidad Complutense de Madrid, Diciembre 1998), de la que Fermín fue mi director, y está dedicado a su memoria. Trabajo financiado por el Proyecto Fauna Ibérica (PB92-0121DGICYT, PB95-0235-DGICYT) y el programa Marie Curie Fellowship de la Unión Europea (Project MCFI-2000-00794).

\section{Referencias}

BARAud, J., 1992. Coleóptères Scarabaeoidea d'Europe. Faune de France et régions limitrophes $\mathrm{N}^{\mathrm{o}} 78$. Société Linnéenne de Lyon et Fédération Francaise des Sociétés de Sciences Naturelles. Lyon. 850 pp.

Brooks, D. R., 1990. Parsimony analysis in historical biogeography and coevolution: Methodological and theoretical update. Systematic Zoology, 39: 14-30.

Browne, D. \& Scholtz, C., 1999. A phylogeny of the families of Scarabaeoidea (Coleoptera). Systematic Entomology, 24: 51-84.

Cheylan, M., 1995. Les reptiles du Paléartique occidental. Diversité et Conservation. Tesis Doctoral. Inédita. Laboratoire de Biogéographie et Ecologie des Vertébrés. Montpellier. 339 pp.

CocA-AвiA, M., 1995. Taxonomía, Filogenia y Biogeografía del género Rhizotrogus (Col., Melolonthidae) en el Mediterráneo occidental. Tesis Doctoral. Inédita. Universidad Complutense de Madrid. 350 pp.

CoOpe, G. R., 1970. Interpretation of Quaternary insect fossils. Annual Review of Entomology, 15: 97-120.

CRACRAFT, J., 1989. Deep-history biogeography: retrieving the historical pattern of evolving continental biotas. Systematic Zoology, 37: 221-236.

DE JONG, H., 1998. In search of historical biogeographic patterns in the western Mediterranean terrestrial fauna. Biological Journal of the Linnean Society, 65: 99-164.

DOADrIO, I., 1990. Phylogenetic relationships and classification of western Palaearctic species of the genus Barbus (Osteichthyes, Cyprinidae). Aquatic Living Resources, 3: 265-282.

DoAdrio, I., Bouhadad, R. \& Machordom, A., 1998. Genetic differentiation and biogeography in Saharan populations of the genus Barbus (Osteichthyes, Cyprinidae). Folia Zoologica, 47: 41-57.

Humphries, C. J., 1992. Cladistic Biogeography. In: P. L. Forey, C. J. Humphries, I. J. Kitching, R. W. Scotland, D. J. Siebert \& D. M. Williams (eds.). Cladistics: A practical course in Systematics. Systematics Association Publications, 10: 137-159.

LACroIX, M., 2000. Hannetons, un monde de diversité. Website: http//hannetons.free.fr. 
LA GreCA, M., 1990. The Insect Biogeography of West Mediterranean Islands. International Symposium on Biogeographical aspects of insularity, Roma: 469-491.

Macey, J. R., Schulte II, J. A., Ananjeva, N. B., Larson, A., Rastegar-Pouyani, N., Shammakov, S. M., \& PAPENFuss, T. J., 1998. Phylogenetic relationships among Agamid lizards of the Laudakia caucasia species group: Testing hypothesis of biogeographic fragmentation and an area cladogram for the Iranian Plateau. Molecular Phylogenetics and Evolution, 10: 118-131.

Morrone, J. J. \& CRisci, J. V., 1995. Historical Biogeography: Introduction to Methods. Annual Review of Ecology and Systematics, 26: 373-401.

Martín-Piera, F. \& SANMARTín, I., 1999. Biogeografía de áreas y biogeografía de artrópodos Holárticos y Mediterráneos. In: A. Melic., J. J. De Haro., M. Méndez \& I. Ribera (eds.). Evolution and Phylogeny of Arthropoda. Boletín de la SEA, 26: 535-560.

Martín-PierA, F. \& Zunino, M., 1985. Taxonomie et biogeographie des Ontophagus du groupe de l'O. ovatus I. (Coleoptera, Scarabaeoidea). Nouvelle Revue d'Entomologie (NS), 2: 241-250.

Nelson, G. J. \& Platnick, N. I., 1981. Systematics and Biogeography: cladistics and vicariance. Columbia University Press. New York. 567 pp.

Oosterbroek, P. \& Arntzen, J. W., 1992. Area cladograms of Circum-Mediterranean taxa in relation to Mediterranean palaeogeography. Journal of Biogeography, 19: 3-20.

PAGE, R. D. M., 1994. Maps between trees and cladistic analysis of relationships among genes, organisms, and areas. Systematic Biology, 43: 58-77.

RonQuist, F., 1996. DIVA v. 1.1. Computer program for MacOS and Win32. Available from

http://www.ebc.uu.se/systzoo/research

RonQuist, F., 1997. Dispersal-Vicariance Analysis: A new approach to the quantification of historical biogeography. Systematic Biology, 46: 195-203.

RonQuist, F., 1998. Phylogenetic approaches in coevolution and biogeography. Zoologica Scripta, 26: 313322.

RonQuist, F., 2002. Parsimony analysis of coevolving species associations. In: R. D. M. Page (ed.). Cospeciation. Chicago University Press. Chicago: 22-64.

Rögl, F. \& Steininger, F. F., 1983. Von Zerfall der Tethys zu Mediterran und Paratethys. Die neogene Palaeogeographie und Palinspastik des zirkum-mediterranen Raumes. Annalen des Naturhistorischen Museums in Wien (Serie A), 85: 135-163.
SAnMartín, I., 1998. Evolución de los Pachydeminae Paleárticos (Col., Scarabaeoidea, Melolonthidae). Tesis Doctoral. Inédita. Universidad Complutense de Madrid. 349 pp.

SANMARTín, I., 2003. Dispersal versus vicariance in the Mediterranean. Historical biogeography of the subfamily Pachydeminae (Coleoptera, Scarabaeoidea, Melolonthidae). Journal of Biogeography, 30: 1-15 (en pernsa).

SAnMartín, I., Enghoff, H. \& Ronquist, F., 2001. Patterns of animal dispersal, vicariance and diversification in the Holarctic. Biological Journal of the Linnean Society, 73: 345-390.

SAnMARtín, I. \& MARTín-Piera, F., 2003. First phylogenetic analysis of the subfamily Pachydeminae (Coleoptera, Scarabaeoidea, Melolonthidae): the Palearctic Pachydeminae. Journal of Zoological Systematics and Evolutionary Research, 41: 2-46.

SAnMartín, I. \& Ronquist, F., 2002. New solutions to old problems: Widespread taxa, redundant distributions, and missing areas in event-based biogeography. Animal Biodiversity and Conservation, 25 (2): 75-93

SAnMartín, I. \& Ronquist, F. (en prensa) Southern Hemisphere Biogeography: Plant vs. Animal patterns. Systematic Biology.

Smith, A. G., Smith, D. G. \& Funnel, B. M., 1994. Atlas of Mesozoic and Cenozoic coastlines. Cambridge University Press. Cambridge. 99 pp.

Thompson, A., 2000. Origins of Arabia. Oriental Press, Dubai. 150 pp.

ViLlalobOS, F. J., 1991. The community structure of soil Coleoptera (Melolonthidae) from a tropical grassland in Veracruz, Mexico. Pedobiología, 35: 225 238.

Villalobos, F. J. \& Lavelle, P., 1990. The soil Coleoptera community of a tropical grassland from Laguna Verde, Veracruz (Mexico). Revue d'Écologie et Biologie du Sol., 27: 73-93.

VoelKer, G., 1999. Dispersal, vicariance, and clocks: historical biogeography and speciation in a cosmopolitan passerine genus (Anthus: Motacillidae). Evolution, 53: 1536-1552.

Waters, J. M., López, J. A. \& Wallis, G. P., 2000. Molecular phylogenetics and biogeography of galaxiid fishes (Osteichthyes: Galaxiidae): Dispersal, vicariance, and the position of Lepidogalaxias salamandroides. Systematic Biology, 49: 777-795. 